\title{
Elaboration and Characterization of Thermal Insulating Material Based on the Synergy of Natural Coconut Husk and Disposable Diaper Pads Fibers
}

\author{
Lenrah Rinna A. Mascariñas ${ }^{1, a}$, Romnick M. Ureta ${ }^{1, b}$, Siegfred M. Mejico ${ }^{1, c}$, \\ Czarina D. Servando ${ }^{1, d}$ \\ ${ }^{1}$ MIMAROPA Regional Science High School, Bansud, Philippines, 5210 \\ alenrah11@yahoo.com, bromnickm.ureta@gmail.com, 'sieg.mejico@gmail.com, \\ ${ }^{d}$ Czarina5sos@gmail.com
}

\begin{abstract}
Keywords: Composite thermal insulators, Coconut husks, Disposable diapers, Solid wastes fibers, Fiber morphology, Thermal conductivity and resistance
\end{abstract}

\begin{abstract}
Materials based on highly porous fibers are now becoming increasingly popular as thermal insulators to fight severely increasing temperatures due to climate change. In this study, fibers from solid waste materials like coconut husk and absorbent pads of used disposable diapers were evaluated for their synergistic effect as thermal insulating material. Dried coconut husk fibers and cleaned used disposable diapers were heated at $150^{\circ} \mathrm{C}-300^{\circ} \mathrm{C}$ for 2 hours and then molded to make pads. Scanning Electron Microscopy and HD digital microscope were used in the analytical imaging of the fibers. Fiber pads from coconut husk fibers, used disposable diapers and their combination, and polyethylene foam (+control) were evaluated for their burning rate based on ASTM D 1992 while thermal conductivity, and thermal resistance were obtained using the testing hot box. The morphology of the coconut husk fibers with used disposable diapers showed thicker and rougher surface with foamy coatings which confirmed porous fiber structure. One-Factor ANOVA showed significant difference among the different set-ups for burning rate $(F=926.506 ; p=0.000)$, thermal conductivity $(F=203.993$; $\mathrm{p}=0.000)$, and thermal resistance $(\mathrm{F}=197.820 ; \mathrm{p}=0.000)$ wherein the set-up for the combination of the coconut husk fibers and used disposable diapers exhibits satisfactory burning rate $(\mathrm{M}=3.268)$, lowest thermal conductivity $(M=0.037)$ and highest thermal resistance $(M=0.273)$. The results affirm that the natural coconut husk fibers and used disposable diapers have synergistic effect for better thermal properties, thus, producing a more effective and safer thermal insulating material.
\end{abstract}

\section{Introduction}

Designing and construction of some of the residential and important public building are done in such a manner that they are least affected by heat due to the severe effect of climate change. The materials which minimize or check the passage of heat through them are called insulators or insulating materials [5]. At present, industrial insulators are manufactured from fiberglass, mineral wool, polystyrene (expanded), and polyurethane (rigid form). Despite the good physical properties of these synthetic insulating materials like low thermal conductivity, good moisture protection and fire resistance, these are very expensive to acquire and these can be hazardous to human health and to the environment. For example, direct exposure to the small particles from fiberglass and glass wool insulation can cause health hazard and respiratory or skin irritation [20]. These problems led to the demand for the utilization of alternative thermal insulation products.

The use of natural thermal insulators derived from fibrous agricultural wastes is becoming of great importance due to their safe application and large contribution to solid waste management. Various sources of these fibrous agricultural wastes are bagasse, coconut husk, corn by-products, cotton wool, sheep wool etc. [13]. Currently, the utilization of fiber-based materials is the most promising for thermal insulation due to their year round availability and low cost. According to Khedari et al. [8], the coconut is one of the natural sources used for the production of insulating materials that meets the economic and environmental requirement due to their low thermal conductivity. 
Meanwhile, estimated 20 billion disposable diapers are added to landfills throughout a particular country each year, creating about 3.5 million tons of waste according to US Environmental Protection Agency (EPA) as cited by CISION PR Newswire (2016). These used disposable diapers decompose in as little as 75 years to as long as 700 years and each year around 82,000 tons of plastic are used during the manufacturing of disposable plastic diaper [22]. The solid waste problem caused by used disposable diapers contributed more in escalating water and land pollution.

In Oriental Mindoro, Philippines, coconut tree is abundant wherein copra making is one of the main and principal livelihood of Mindorenos and according to the 2016 report of the provincial solid waste management board, about $45 \%$ of the solid waste generated in the province are plastic products and $50 \%$ of which are the accumulated used disposable diapers which caused serious clogging on water pathways particularly in urban areas.

In this context, this work aims to study the feasibility of using coconut husk fibers mixed with the absorbent pad of used disposable diapers and determine their synergistic effect as thermal insulation medium. In addition, in this study hypothesizes no significant difference among the different set-ups such as the combination of coconut husk fibers and used disposable diapers and their individual applications, and the positive control as thermal insulators in terms of burning rate, thermal conductivity, and thermal resistance.

In this study, an insulating material was developed from the combination of coconut husk and absorbent pads of used disposable diapers and determine its thermal properties such as thermal conductivity (lamda) and thermal resistance $\left(\mathrm{R}_{\text {th }}\right)$ an calibrated hot box are used according to the "Thermal Testing of Building Insulation Materials" [3]. Other parameters like, burning rate ( $\mathrm{mm} /$ minute) and fiber morphologies were also evaluated.

The development of the new insulation medium from the natural fibers of coconut husk and fibrous absorbent pads from used disposable diapers is a new leap in environmental engineering since no previous researches studied their possible synergistic effect for better thermal insulation capacity. This study also addresses the problem in solid waste management, promotes recycling, and could even provide new source of income for local farmers.

\section{Materials and Methods}

\section{Collection of materials}

Coconut husk fibers were gathered from the nearby coconut farm in Ipil, Bongabong, Oriental Mindoro and were authenticated, and verified by the National Museum-Botany Division, Padre Burgos, Ermita, Manila, Philippines. On the other hand, used disposable diapers were collected at the landfills and household trash bins in the Municipality of Bongabong, Oriental Mindoro.

On the other hand, the equipment used for the experimentation were hot plate, glass beakers, iron sheet molder, 100-watt light bulb, old CPU fans, stopwatch as timer, thermal reflective aluminum foil bubble or polyethylene aluminum foil foam insulation sheet (in surrounding the hot box), digital balance, L2T-0205 brazing blow torch, a 200x-1600x digital microscope with 0 200mm focus range, and a TC-3200 digital thermometer with Type K-8mm thermocouple probes.

\section{Preparation of the Used Disposable Diapers and Coconut Husk Fibers}

Disposable diapers consist of a plastic outer layer with integral fastenings and a core of absorbent materials with a protective top layer. The nappy core is composed of fluff pulp (cellulose fiber) and a water-absorbent polymer, sodium polyacrylate (SAP). The top layer is made up of a nonwoven polymer-based material.

To eliminate foul odors, foreign dirt and to at least sterilize the material, the collected used disposable diapers were initially washed with some detergent and then treated with $6 \%$ sodium hypochlorite and rinsed in a continuous flowing tap water. The diaper pads were then disinfected via autoclave at $120^{\circ} \mathrm{C}$ within 30 minutes. The pads were then sundried until fully dehydrated to reduce the moisture. The fully dehydrated and solidified form of the used disposable diapers were dissected 
using scissors to obtain the fibrous material. All the collected fibers from the absorbent pads of diapers were placed in a glass container and set aside for future use.

On the other hand, the gathered natural coconut husk fibers were sundried and manually sorted to remove foreign fibers and dirt. The cleaned fibers were carefully shredded to produce thin/narrow type of fibers.

\section{Production of the Thermal Insulating Material}

Fifty (50) grams of absorbent pad of used disposable diaper was placed in a 1-liter glass beaker and allowed to absorb $1000 \mathrm{ml}-3000 \mathrm{ml}$ of distilled water. After the water was fully absorbed and displayed gel-liked appearance, the used disposable diapers were divided into half and placed into two separated 1-liter glass beakers for more convenient stirring and mixing process. Meanwhile, fifty (50) grams of coconut husk fibers was mixed to the used disposable diapers. The mixtures were heated using a hot plate at $150^{\circ} \mathrm{C}-300^{\circ} \mathrm{C}$ until gel like formation and enough viscosity of the diaper absorbent pad are observed and the natural dye (red to reddish brown) of the coconut husk fibers appeared. The mixture was continuously stirred until more viscous state. The mixtures were transferred into a $300 \mathrm{~mm} \times 300 \mathrm{~mm}$ iron sheet molder with $10 \mathrm{~mm}$ thickness and sundried for seven days until total dehydration is attained. On the other hand, 100 grams of pure coconut husk fibers and pure absorbent pad of diaper were also molded in the same size.

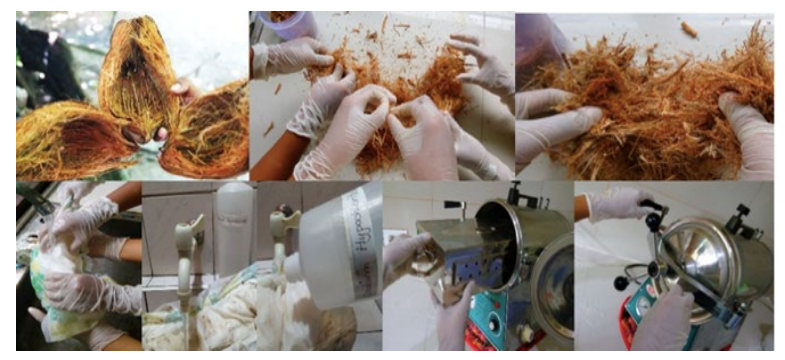

Figure 1. Preparation of the raw coconut husk fibers and cleaning the disposable

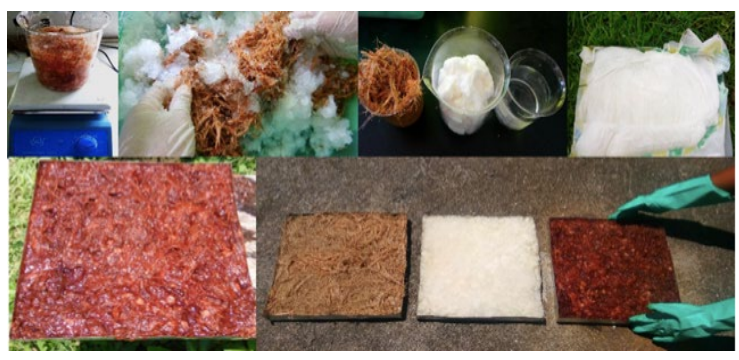

Figure 2. Mixing and molding the synergized insulating material together wiht the other set-ups.

There were four set-ups in the study:

Set-up 1: $50 \mathrm{~g}$ absorbent pad of diaper with $50 \mathrm{~g}$ coconut husk fibers $(1=300 \mathrm{~mm} ; \mathrm{w}=300 \mathrm{~mm} ; \mathrm{h}=10 \mathrm{~mm})$ Set-up 2: $100 \mathrm{~g}$ of coconut husk fibers $(1=300 \mathrm{~mm} ; \mathrm{w}=300 \mathrm{~mm} ; \mathrm{h}=10 \mathrm{~mm})$

Set-up 3: $100 \mathrm{~g}$ of absorbent pad of used disposable diaper $(\mathrm{l}=300 \mathrm{~mm} ; \mathrm{w}=300 \mathrm{~mm} ; \mathrm{h}=10 \mathrm{~mm})$

Set-up 4: Positive Control (polyethylene foam insulation sheet) $(1=300 \mathrm{~mm} ; \mathrm{w}=300 \mathrm{~mm} ; \mathrm{h}=10 \mathrm{~mm})$.

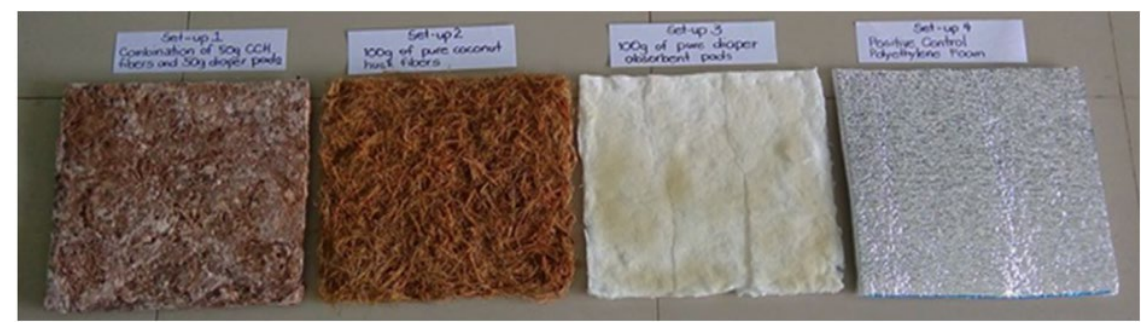

Figure 3. The different set-ups in the study.

Fiber Morphology Evaluation

The evaluation of the fiber morphology of the constructed fiber pads was done using JSM-5310 Scanning Electron Microscope and Digital HD Microscope. In doing the SEM, coconut husk fibers with and without used disposable diaper and the pure diaper pad were cut $4-5 \mathrm{~mm}$ in length and carefully attached on the small metal platform holder using carbon tape. Possible dirt and dust on the sample were removed using a bulb syringe aspirator. Before the actual SEM observation, the 
specimens underwent auto fine coating using JEOL JFC-1200 Fine Coater. Three frames of micrographs were generated for each specimen given at different magnifications. On the other hand, the actual prepared pads were used in HD digital microscope for further surface evaluation.

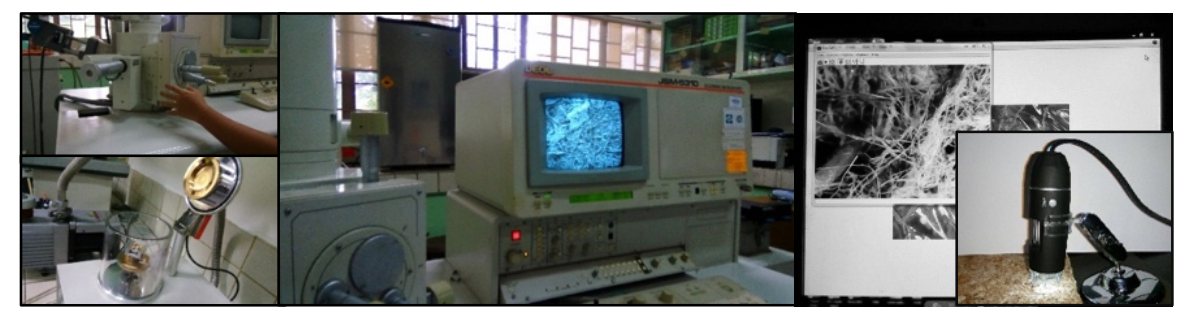

Figure 4. Scanning electron microscopy (SEM) and HD digital microscope imaging of the insulting material.

\section{Burning Rate Test}

The calculation of the actual burning rate confirms the extent of the possible flame tolerance of the thermal insulation medium to ensure safety factor.

The standard test procedure applied in this test is a modification of ASTM D 1992 test for the flammability of fiber materials and plastics. The flame test was conducted in a chamber $381 \mathrm{~mm}$ long, $203.2 \mathrm{~mm}$ thick and $355.6 \mathrm{~mm}$ high. The chamber is equipped with a glass observation window, a closable opening for the insertion of specimen, a hole to accommodate the L2T-0205 brazing blow torch as burner and ventilation holes on the side. The gas flame in the brazing blow torch was monitored to have $1000^{\circ} \mathrm{C}-1200^{\circ} \mathrm{C}$ using the TC-3200 digital thermometer connected to a Type-K $8 \mathrm{~mm}$ thermocouple probe. A $100 \mathrm{~mm} \times 100 \mathrm{~mm}$ specimen was put in the holder inside the chamber. All the specimens were tested with five (5) replications. The rate, at which the flame burns down the specimen for a distance of $11279.4 \mathrm{~mm}$, was then calculated by the formula:

$$
\text { Burning Rate }=60 x \frac{\text { Distance burned }(\mathrm{mm})}{\text { Time }(\text { seconds })}
$$

\section{Heat Transfer Testing Procedures}

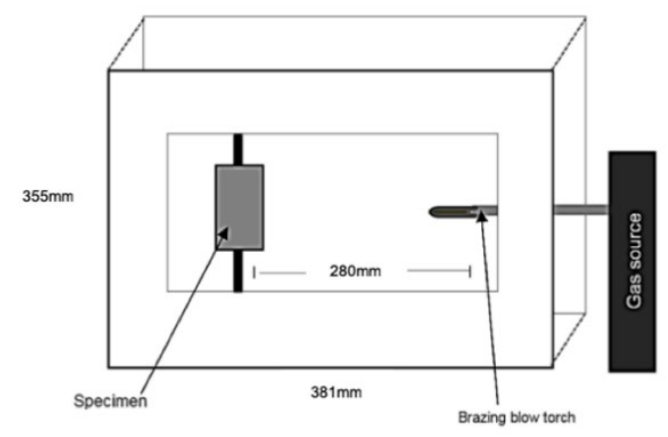

Figure 5. The Burning Rate Chamber as per ASTM.

The thermal conductivity $(\lambda)$ and thermal resistance $\left(\mathrm{R}_{\mathrm{th}}\right)$ of the thermal insulation medium were evaluated and calculated using the testing hot box with slight modifications following the specifications provided by Frawley and Kennedy [3] in their "Thermal Testing of Building Insulation Material'”. 


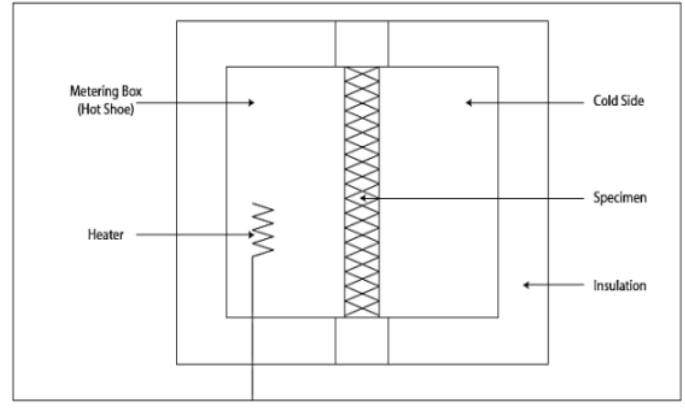

Figure 6. The internal diagram of the testing hot box [3].

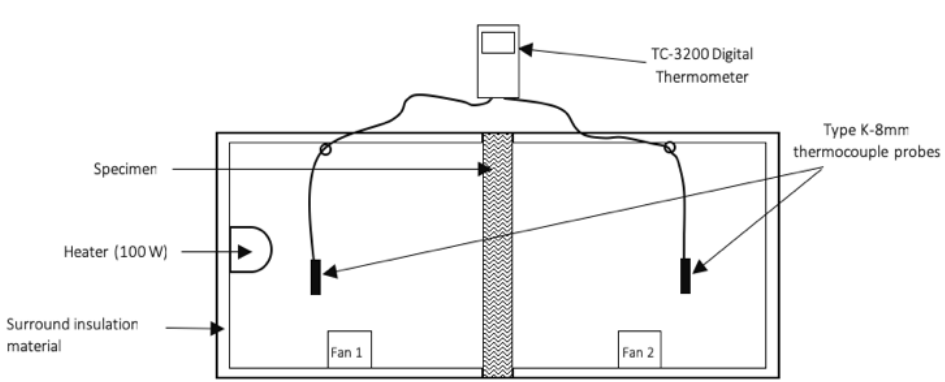

Figure 7. The actual diagram for testing hot box set-up.

The testing hot box contained of two chambers, the hot side and the cold side. The walls were insulated to keep the heat losses to the environment at a minimum as major losses would lead to inaccuracies in measurements.

The test specimen was fixed into a surround panel that will be located between the two chambers. The thickness of the surround panel varies according to the standard to which the hot box is built. Air was circulated in both chambers to prevent hot or cold spots occurring in the chambers. The velocity of the air moving over both sides of the test specimen affects the heat transfer through the test specimen and this was accounted for the calculations. Temperatures were measured within 10 minutes using TC-3200 Digital Thermometer that was connected to a Type K-8mm thermocouple probe. Observation for the thermal properties of the specimen was made with 10 trials using heat flow of 100 watts with the average temperature range of $90^{\circ} \mathrm{C}-93^{\circ} \mathrm{C}$ (within 10 minutes) [10].

The thermal conductivity was calculated using the formula, $\lambda=\frac{Q L}{A \Delta T}$ where $\lambda$ is the thermal conductivity, $\mathrm{Q}$ is the heat transfer in watts, $\mathrm{L}$ is the thickness, $\mathrm{A}$ is the area, and $\Delta T$ is the change in temperature in Kelvin. On the other hand, the thermal resistance $\left(\mathrm{R}_{\mathrm{th}}\right)$ which is the function of the actual thickness of the material and the thermal conductivity $\lambda$ was calculated using $\mathrm{R}_{\mathrm{th}}=\frac{L}{\lambda}$ where $\mathrm{L}$ is the actual thickness of the material expressed in meters [6].

\section{Statistical Analysis}

The thermal conductivity, heat and thermal resistance of the thermal insulation medium made from the combination of the coconut husk and absorbent pad of used disposable diapers and their individual formulations were compared via One-Factor Analysis of Variance (ANOVA) wherein pvalue of $\mathrm{p}<0.05$ was accepted statistically significant. To further test the validity of the results, posthoc test was used with a significance threshold of 0.05 . 


\section{Results}

A. Fiber Morphology via Scanning Electron Microscopy (SEM)
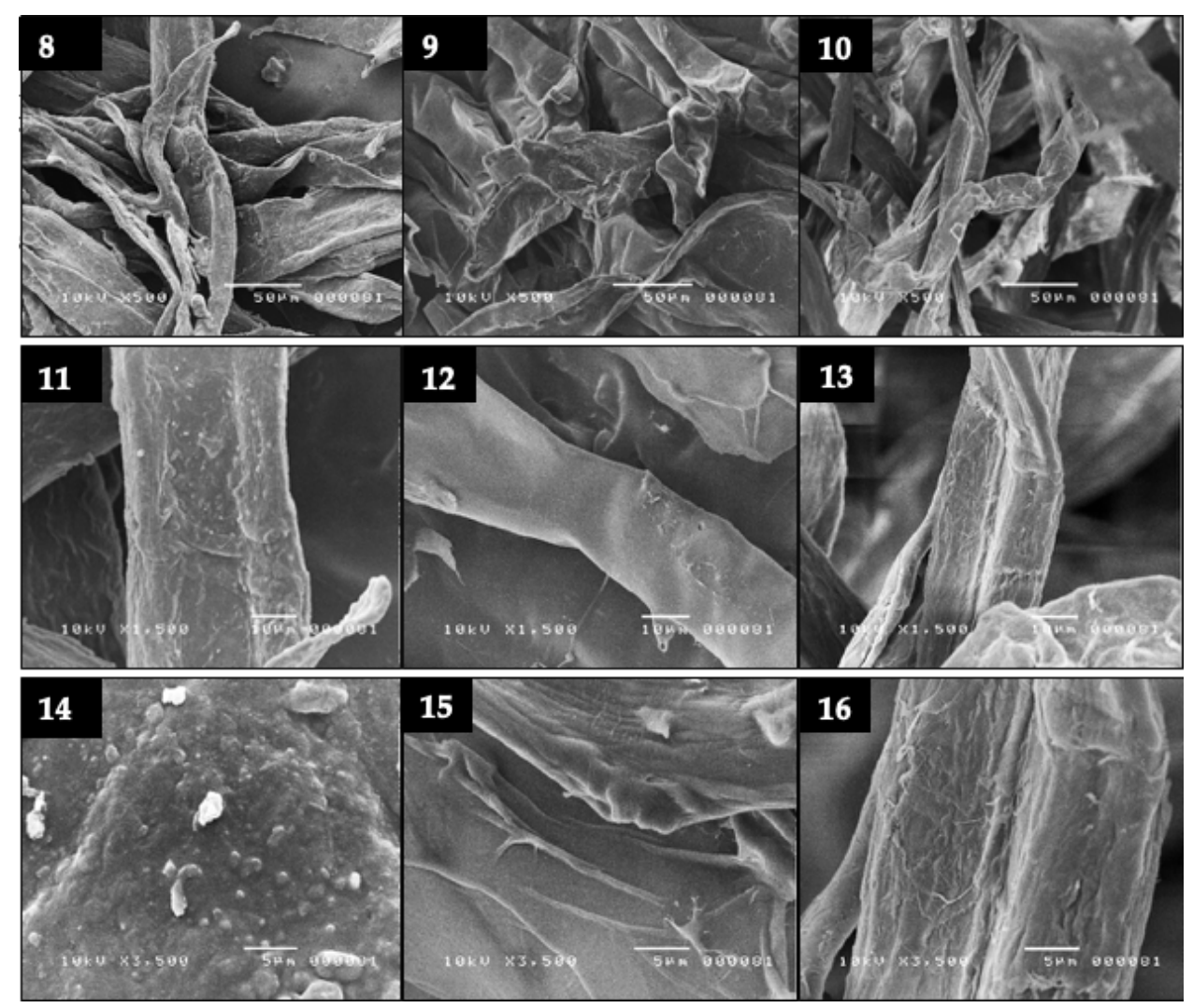

Figures 8-16. SEM micrographs of the coconut husk fibers with absorbent pads of used disposable diapers (Figs. 8, 11, and 14), pure coconut husk (Figs. 9, 12, and 15), and pure diaper pads (10, 13, and 16) at 500x and 1,500x, and 3,500x magnifications.

The results of the morphology evaluation of the coconut husk fibers and absorbent pads of used disposable diapers and their combination via Scanning Electron Microscopy (SEM) are shown in Figs. 8-16 at 500x, 1,500x, and 3,500x magnifications respectively. The generated analytical imaging of the combined coconut husk fiber used diapers appeared to have a different structure compared to their individual forms. At 1500x and 3500x magnifications, a rougher surface was evident to the coconut husk fiber with used disposable diaper pads (Figs. 11 and 14). The dominance of foamy coverings on the surface of the coconut husk fibers in Figs. 8, 11 and 14 were due to the presence of superabsorbent polymers (SAPs) among disposable diapers [16]. The SAPs has a very high porosity since it functions as general absorbent among diapers as evident in Figs. 10, 13, and 16 wherein white foamy surfaces are shown. On the other hand, the raw coconut husk fibers (Figs. 9, 12, and 15) exhibited inconsistent roughness in the surface. A rooted but clean with slightly smooth structure was then revealed in the surface of the pure coconut husk fiber in Fig. 13 at 3,500x magnification. Meanwhile, the gel-like coating on the surface of the fiber of coconut husk in Fig. 11 affirmed the porous surface which produces good fiber bonding among the fiber strands. Thicker and rougher fibers results to lower thermal conductivity [6].

Porous materials often exhibited severely rough surface structure with the existence of some impurities [12]. Porosity of the fibrous materials are often explained and exhibited by their texturized, macro-cellular and foamy surface [4] as evident to the micrographs of the pure diaper pad (Figs. 10, 13 and 16) and the combination of coconut husk fibers and used disposable diapers (Figs. 8, 11 and 14). Effective and efficient natural thermal insulating materials are those fibers which possess high porosity [5]. Based on its morphology, the combination of coconut husk fibers and absorbent pads of the used disposable diapers affirms porosity (Figs. 8, 11, and 114). This further testifies that their combination may have a very promising thermal insulating performance. 


\section{B. Fiber Morphology via HD digital light microscope}

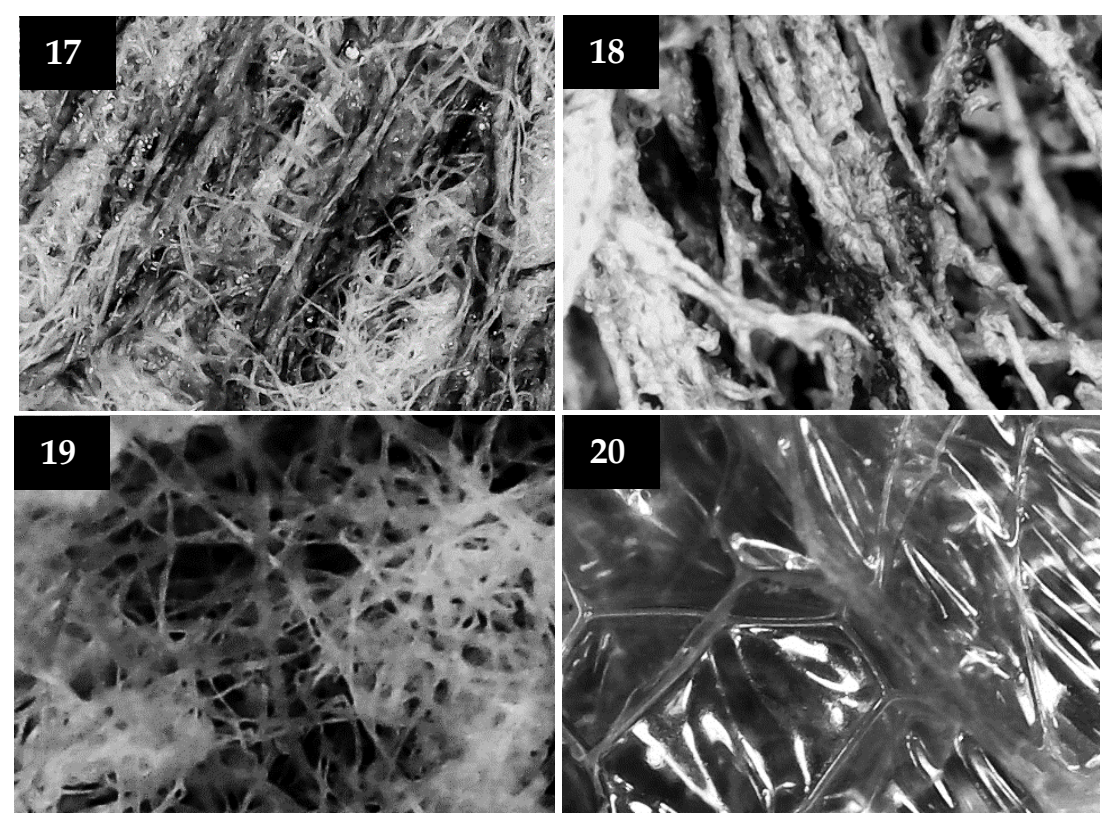

Figures 17-20. Digital imaging of the coconut husk fibers with absorbent pads of used disposable diapers (Fig. 17), raw coconut husk (Fig. 18), pure absorbent pad of used disposable diaper (Fig. 19), and the polyethylene aluminum foil foam insulation sheet (Fig. 20) given at 200x magnification.

The fibrous structure of the actual thermal insulation materials from the coconut husk fibers, absorbent pad of the used disposable diapers, their combination and the positive control are shown in Figs. 17-20. The figures also depicted how the structure of the raw fibers of the coconut husk improved after mixing in the absorbent pads of the used disposable diapers.

It can be deduced from the image in Fig. 18 that raw fibers of the coconut husk have divisive fiber placement despite of its solidified and rough fiber surface. This condition of raw fibers was then improved by adding the absorbent pads of used disposable diapers as shown in Fig. 17. The longitudinal compartments between fiber strands were resolved by the fibers of the absorbent pads of the diapers. The combination of the coconut husk fibers and fibrous absorbent pad of diapers produced fiber structure with good thermal bonding. Meanwhile, the fibrous absorbent pad of the diapers is shown in Fig. 19. The fibers in Fig. 19 are thinner with large gaps from one another since the fibrous absorbent pads of disposable diapers are made from cotton with superabsorbent polymers [16]; so, expected that finer strands are to be observed. The combination of the coconut husk fibers and the fibrous absorbent pads of diapers make the insulation medium more porous and compact since the SAPs of diapers served as coatings after the gel-liked formation during the heating process. The finer fiber strands of the diaper absorbent pad filled the longitudinal compartments between coconut husk fiber strands making the insulation medium more porous. Porous materials are better thermal insulators which is directly associated with lower thermal conductive values [17]. On the other hand, a glossy, smooth, with soft-rooted structure were observed for the surface of the polyethylene foam insulation in Fig. 20. Divisive strands were not evident on Fig. 18 due to the fact that it is made from plastic. The positive control tend to have a foamy surface because of its softer surface. According to Luo and $\mathrm{He}$ [21], these surface characteristics may have low thermal properties but highly susceptible to fire. 


\section{Burning Rate Test}

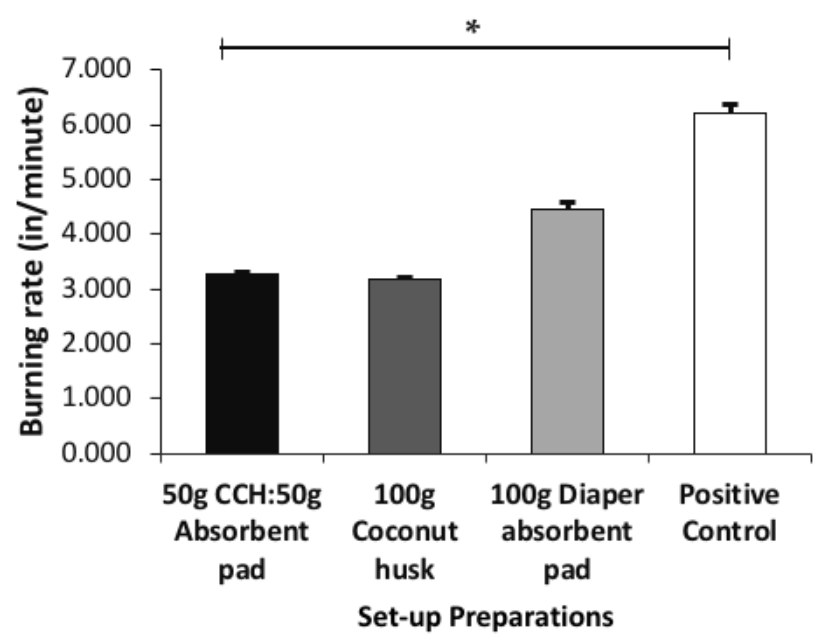

Figure 19. Average burning rate of the four set-ups evaluated with five replications. ${ }^{*} p<0.05$ was used to show significant difference among the set-up preparations using One-Factor ANOVA.

To distinguish safety factor and flame tolerance of the materials used as thermal insulation medium, the test was conducted based on the specifications from ASTM D 1992 which considers burning distance. Burning rates in this test testify higher accuracy compared to other flame tolerance tests which subjected their specimens to the direct exposure to the flame.

Based on Fig. 19, One-Factor ANOVA results revealed that there is significant difference among the set-ups in terms of the burning rate $[F(3,16)=926.506, p=0.000]$ wherein it was determined through Post Hoc analysis that significant difference occurred at the following mean pairings of the given set-ups such as: set-up 2 vs set-up $3(\mathrm{M}=3.185 ; \mathrm{M}=4.462)$; set-up 3 vs set-up 4 $(\mathrm{M}=4.462 ; \mathrm{M}=6.218)$ and set-up 1 vs set-up $3(\mathrm{M}=3.268 ; \mathrm{M}=4.462)$; set-up 1 vs set-up $4(\mathrm{M}=3.268$; $M=6.218)$; and set-up 2 vs set-up $4(M=3.185 ; M=6.218)$. The results testify that among the set-ups, the pure coconut husk fibers have the lowest burning rate followed by the combination of the coconut husk fibers and diaper absorbent pads while the control set-up which is the application of polyethylene foam insulation sheet has the highest burning rate. The results further imply that the combination of the natural fibers of coconut husk and the diaper absorbent pads affirms insusceptibility to fire.

The coconut husk fibers have a good flame tolerance compared to other commercial foam insulation [10]. In this study, the coconut husk fibers have improved their insusceptibility to flames by adding the fibrous diaper absorbent pads. The obtained result of 3.268 inches/minute for the combination of coconut husk and diaper absorbent pads is considered to be satisfactory as per specifications presented in ASTM D 1992 (National Materials Advisory Board, National Academy of Sciences, 1980). According to Wang et al. [17], fibrous materials are considered to have high porosity and this porosity is directly associated with higher flame tolerance which means a lower burning rate. The fiber morphology via SEM (Figs. 8, 11, and 14) of this study supports the claim of porous structure of the coconut husk fibers with diaper absorbent pads. The results are also parallel to that of Ganiron [5] wherein the coconut husk fibers were mixed to polypropylene material. This means that the utilization of the natural coconut husk fibers when combined to other fibrous material often gives a higher tendency of having good flame tolerance. So, the synergy of the coconut husk and the diaper absorbent pads suggests lower burning rates which assures a safer thermal insulating material.

In contrast, the results also revealed that the pure diaper absorbent pads appeared to have higher burning rate of 5.08 inches/minute. This is because, specimens had $0 \%$ moisture the time they underwent burning rate test and still the diaper absorbent pads are made from cotton fibers [19]. During this condition, the pads became more vulnerable to fire. On the other hand, high burning rate of the polyethylene is due to its capacity to initiate ignition at approximately starting time of 6 seconds after exposure to direct flame [21]. It is a plastic product made by reacting molecules of ethylene gas into long polymer chains. In addition, polyethylene foams have melting point at $>90^{\circ} \mathrm{C}$, and there is potential for fire or explosion if it is not fully degassed. 
D. Thermal Conductivity

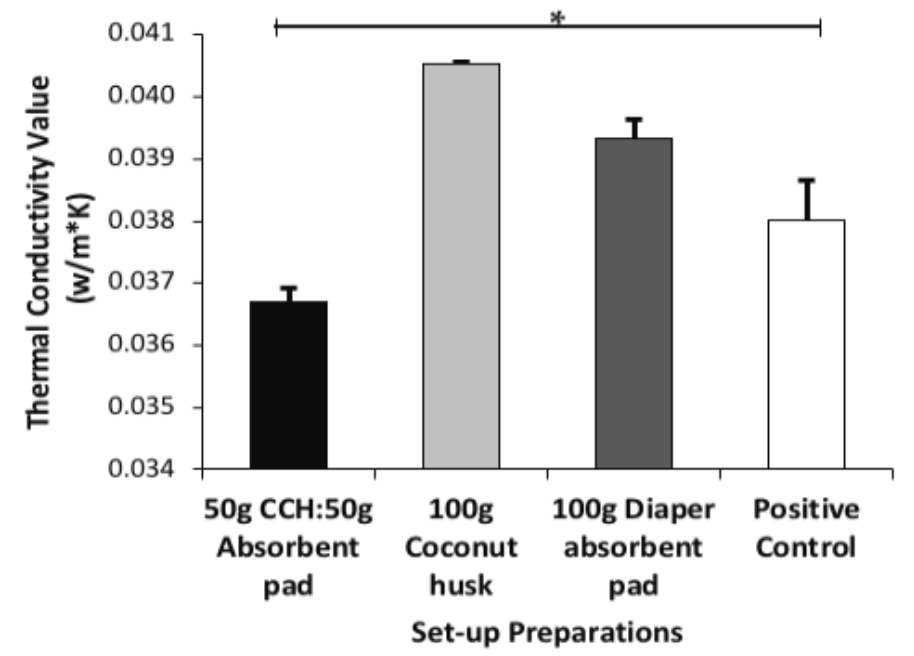

Figure 20. Mean thermal conductivity value $(\lambda)$ of the four set-ups evaluated at a 10 -minute testing with ten trials. ${ }^{*} \mathrm{p}<0.05$ was used to show significant difference among the set-up preparations using One-Factor ANOVA.

In the conducted study, thermal conductivity values $(\lambda)$ were obtained using a ten-minute testing with ten trials using the calibrated hot box. One-Factor ANOVA results revealed that there is a significant difference on the actual thermal conductivity $(\mathrm{W} / \mathrm{m} \cdot \mathrm{K})$ of the different set-up preparations $[F(3,36)=203.993, p=0.000]$ at $p<0.05$ level of significance. From the results, the set-up for the combination of the coconut husk fibers and absorbent pads of used disposable diapers has the lowest thermal conductivity value $(\mathrm{M}=0.037 \mathrm{~W} / \mathrm{m} \cdot \mathrm{K})$ while the set-up of pure $100 \mathrm{~g}$ of natural coconut husk fibers has the highest thermal conductivity $(\mathrm{M}=0.041 \mathrm{~W} / \mathrm{m} \cdot \mathrm{K})$.

Post Hoc analysis using Scheffe shows that the mean values presented in Fig. 20 with attached asterisk signs have significant difference result $(\mathrm{p}<0.05)$. Significant mean differences are shown in all mean pairings such as: set-up 1 vs set-up $2(\mathrm{M}=0.037 ; \mathrm{M}=0.041)$; set-up 2 vs set-up $3(\mathrm{M}=0.041$; $\mathrm{M}=0.039)$; set-up 3 vs set-up $4(\mathrm{M}=0.039 ; \mathrm{M}=0.038)$; set-up 1 vs set-up $3(\mathrm{M}=0.037 ; \mathrm{M}=0.039)$; setup 2 vs set-up $4(\mathrm{M}=0.041 ; \mathrm{M}=0.038)$; and set-up 1 vs set-up $4(\mathrm{M}=0.037 ; \mathrm{M}=0.038)$. Among the prepared set-ups for thermal insulation medium, set-up 1 or the combination of the coconut husk fibers and absorbent pads of used disposable diapers significantly has the lowest thermal conductivity value $(\lambda)$. Lower conductivity values for insulating materials is inversely proportional to the thermal insulating capacity. Thus, the lower the thermal conductivity the higher the insulation performance. The result then testify that the synergy of the coconut husk fibers and the absorbent pads of used disposable diapers performed better as thermal insulation medium.

Meanwhile, natural coconut husk fibers specimens tested vertically and horizontally in a thermal conductivity apparatus based on ASTM norm C518 had a thermal conductivity value of $0.049 \mathrm{~W} / \mathrm{m} \cdot \mathrm{K}$ [15]. This is closer to the obtained results of $0.041 \mathrm{~W} / \mathrm{m} \cdot \mathrm{K}$ in the current study for the natural coconut husk fibers wherein a calibrated hot box was used with a hot chamber and cold chamber which is a more advanced control system and test procedure with higher accuracy with capability of measuring the thermal properties of a wider range of building materials [3]. On the other hand, Agham Rahul [23] investigated the thermal conductivity of the combination of coconut husk and polyurethanes as alternative insulating material and obtained $0.036 \mathrm{~W} / \mathrm{m} \cdot \mathrm{K}$ as thermal conductivity value while in the current study, the synergy of the coconut husk and fibrous diaper absorbent pads has thermal conductivity of $0.037 \mathrm{~W} / \mathrm{m} \cdot \mathrm{K}$. This suggests that the coconut husk tend to have an improved thermal conductivity performance when combined to other fibrous materials. But what makes the synergy of the coconut husk fibers and diapers absorbent pads more advantageous is that the absorbent pads from diapers are made from cotton with SAPs or hydrogels which have been considered as non-hazardous substance or mixture by Breckland Scientific Safety Data Sheet (SDS) 
based on Regulation (EC) No.1907/2006 Article 31. On the other hand polyethylene aluminum foil foam insulation sheets have also a lower thermal conductivity. In fact, these insulation materials are made from plastics known not to occur naturally which is not environmental friendly. Results then give the implication that coconut husk fibers and diapers absorbent pads clearly exhibit synergistic effect when it comes to lower thermal conductivity value which is a good indicator of better and safer thermal insulator.

\section{E. Thermal Resistance}

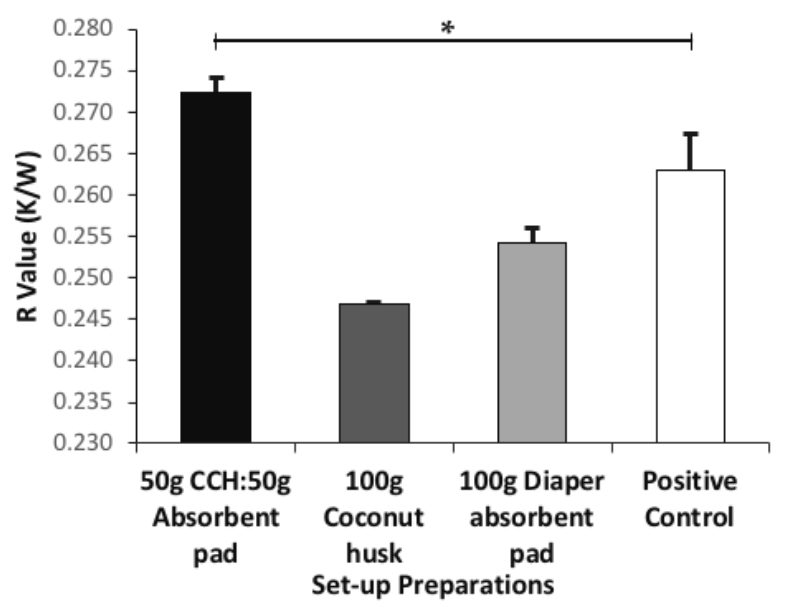

Figure 21. Average thermal resistance $\left(R_{\text {th }}\right)$ value of the three set-ups evaluated at a 10-minute testing with ten trials. ${ }^{*} \mathrm{p}<0.05$ was used to show significant difference among the set-up preparations applying One-Factor ANOVA.

The thermal resistance is the function of the actual thickness of the material and the thermal conductivity $\lambda[6]$.

After evaluating the temperatures in the designed hot box, the thermal resistance $\left(\mathrm{R}_{\mathrm{th}}\right)$ values were calculated. Based on Fig. 21, one-factor ANOVA results showed that there is significant difference on the obtained thermal resistance $\left(\mathrm{R}_{\text {th }}\right)$ values of the prepared set-ups for thermal insulation medium $[\mathrm{F}(3,36)=197.820, \mathrm{p}=0.000]$ at $\mathrm{p}<0.05$ level of significance wherein via Post Hoc analysis it was found that all the mean pairings exhibited significant difference such as set-up 1 vs set-up $2(\mathrm{M}=0.273 ; \mathrm{M}=0.247)$; set-up 2 vs set-up $3(\mathrm{M}=0.247 ; \mathrm{M}=0.254)$; set-up 3 vs set-up 4 $(\mathrm{M}=0.254 ; \mathrm{M}=0.263)$; set-up 1 vs set-up $3(\mathrm{M}=0.273 ; \mathrm{M}=0.254)$; set-up 2 vs set-up $4(\mathrm{M}=0.247$; $\mathrm{M}=0.263)$; and set-up 1 vs set-up $4(\mathrm{M}=0.273 ; \mathrm{M}=0.263)$. The obtained results reveal that set-up 1 which is the synergy of the coconut husk fibers and the fibrous diaper absorbent pads has the highest thermal resistance which is because of the improved fiber structures of the coconut husk fibers due to the addition of diaper absorbent pads which is known to have SAPs which exhibit foamy surface of rougher and thicker fibers.

High thermal resistance assures the effectivity of the developed thermal insulation medium [15]. The obtained value of $0.273 \mathrm{~K} / \mathrm{W}$ for the synergy of the coconut husk fibers and diaper absorbent pads is associated with its porous fiber structure. The superabsorbent polymers (SAPs) content of the diaper absorbent pads [19] served as coatings to the natural fibers of the coconut husk and made the fibers thicker and rougher. According to Wang et al. [17], rough surfaced-materials are highly porous which has higher thermal resistance resulting to a better thermal insulation performance. This further implies that synergistic effect is evident for the natural coconut husk fibers and fibrous used disposable diaper pads.

Meanwhile, the raw coconut husk fibers have the lowest thermal resistance compared to the other set-ups. This is maybe because of the susceptibility of the coconut husk fibers into flame retained unlike the condition of the two set-ups wherein there is a direct (set-up 3) and partial (set-up 1) presence of porous disposable diaper absorbent pads. Superabsorbent polymers (SAPs) found in 
the disposable diaper absorbent pads can hold over 600 times their weight in water (Gillman, 2008). So, having the combination of two porous fibers could result in an evident synergistic effect of higher thermal resistance. This proves that the synergy of the coconut husk fibers and fibrous diaper absorbent pads could perform as an effective thermal insulating material.

\section{Conclusion}

Based on the number of evaluations conducted, this study has met its objectives. The study was able to determine a significant difference among the four set-ups in terms of burning rate, thermal conductivity and thermal resistance. The set-up for the combination of the coconut husk fibers and used disposable diaper absorbent pads has the lowest thermal conductivity and satisfactory burning rate with highest thermal resistance which is supported by its morphology which exhibits a more improved fiber structure containing foamy white coatings due to the presence of SAPs resulted to a rougher and thicker fibers. Moreover, the polyethylene foam insulation sheet has also a lower thermal conductivity but with higher burning rate which could result in higher vulnerability to flames. This further affirms that the natural coconut husk fibers and used disposable diaper absorbent pads have synergistic effect for better thermal insulating properties, thus, producing a more effective and safer thermal insulation medium.

\section{Conflict of Interest}

The authors declare no conflict of interest. The funding sponsors had no role in the design of the study; in the collection, analyses, or interpretation of data; in the writing of the manuscript, and in the decision to publish the results.

\section{Acknowledgments}

The researchers would like to express their sincerest gratitude to Mr. Juanito Foronda and Mr. Reynaldo Coria of the De La Salle University-Physics Department; the colleagues at MIMAROPA Regional Science High School are also acknowledged.

\section{References}

[1] R.D. Agham, Performance of Coconut Husk Mixed Polyethylene alulinum foil foam insulation sheet as an Alternative Insulating Material, International Journal of Engineering and Innovative Technology. 2(9) (2013) 100-104.

[2] S. Ersoy, H. Kucuk, Investigation of industrial tea-leaf-fiber waste material for its sound absorption properties, Applied Acoustic. 70 (2009) 215-220.

[3] E. Frawley, D. Kennedy, Thermal Testing of Building Insulation Materials, Engineers Journal. 61(9) (2007) 552-558.

[4] M. Fukushima, Y. Yoshizawa, Fabrication and morphology control of highly porous mullite thermal insulators prepared by gelation freezing route, Journal of the European Ceramic Society. (2015) 1-7.

[5] T. Ganiron, Investigation on the use of Coco Coir Polypropylene as Thermal Insulator. International Journal of Advanced Sciences and Technology. 59 (2013) 13-26.

[6] S.K. Ghosh et al., Study on Potential Application of Natural Fibre Made Fabrics as Thermal Insulation Medium. American International Journal of Research in Science, Technology, Engineering \& Mathematics. 16(203) (2016) 8-13.

[7] J. Gillman, The Truth about Garden Remedies, Portland London: Timber Press, 2008. 
[8] J. Khedari et al., New low-cost insulation particleboards from mixture of durian peel and coconut coir, Building and Environment. 39 (2004) 59-65.

[9] M. Lou, Y. He, Combustion Behaviour of Polyethylene aluminum foil foam insulation sheets under depleted Oxygen Environment. Center for Environmental Safety and Risk Engineering.CSIRO Building Construction and Engineering, 220.

[10] G. Manlapas, J. Banaldia, Drinking Straw and Coco Coir Fiber as Thermal Insulator: A Technology Innovation. Imperial Journal of Interdisciplinary Research (IJIR). 2(11) (2016) 1296-1301.

[11] National Materials Advisory Board-National Academy of Sciences (1980). Fire Safety Aspects of Polymeric Materials. Land Transportation Vehicles. Technomic Publishing Co.,Inc., 8,15.

[12] A. Oushabi et al., Natural thermal-insulation materials composed of renewable resources: characterization of local date palm fibers (LDPF), J. Mater. Environ. Sci. 6(12) (2015) 33953402 .

[13] S. Pujari, T. Venkatesh, H. Seeli, Experimental Investigations on Thermal Conductivity of Fenugreek and Banana Composites. The Institution of Engineers, 2017.

[14] J.M. Robine et al., Death toll exceeded 70,000 in Europe during the summer of 2003.C R Biol. 331(2) (2008) 171-178.

[15] N.J. Rodriguez et al., Assessment of coconut fibre insulation characteristics and its use to modulate temperatures in concrete slabs with the aid of a finite element methodology, Energy and Buildings. 43 (2011) 1264-1272.

[16] R. Sanchez-Orozco et al., Valorization of superabsorbent polymers from used disposable diapers as soil moisture retainer. International Journal of Research - Granthaalayah. 5(4) (2017) 105-117.

[17] Z. Wang et al., Porous mullite thermal insulators from coal gangue fabricated by a starch-based foam gel-casting method. Australian Ceramic Society, (2017) DOI: 10.1007/s41779-017-00359.

[18] H. Yang, J. Dae, H.J. Kim, Rice straw-wood particle composite for sound absorbing wooden construction materials, Bioresource Technology. 86 (2003) 117-121.

[19] J. Hower, S. Rochefort, E. Momsen, Disposable Diapers and SAP Activity, 2004. Available: https://engineering.oregonstate.edu/momentum/k12/apr04/index.html.

[20] M. Chikhi, Mourad et al., Experimental investigation of new biocomposite with low cost for thermal insulation, Energy and Buildings. 66 (2013) 267-273.

[21] M. Luo, Y. He, V. Beck, Application of field model and two-zone model to flashover fires in a full-scale multi-room single level building, Fire Safety Journal. 29(1) (1997) 1-25.

[22] Patty Hahne, Are Biodegradable Diapers Really Better For The Environment?, 2013. [Online]. Available: https://www.booneyliving.com/1764/are-biodegradable-diapers-really-better-forthe-environment/.

[23] Agham Rahul D., State of the art insulation materials: a review, International Journal of Engineering and Innovative Technology. 2(6) (2012) 97-102. 